\title{
MOTIVAÇÃO ORGANIZACIONAL: FATORES PRECURSORES DA MOTIVAÇÃO DO COLABORADOR
}

\author{
ORGANIZATIONAL MOTIVATION: FACTORS THAT \\ CONTRIBUTE TO THE EMPLOYEE'S MOTIVATION
}

\author{
Marco Ferreira Ribeiro ${ }^{1}$ \\ Clotilde Passos ${ }^{2}$ \\ Paulo Pereira ${ }^{3}$
}

\begin{abstract}
Resumo: Este estudo visa abordar a temática da motivação organizacional, a evolução histórica da perceção da motivação por parte da gestão, a sua relação com a satisfação no trabalho, as diferentes teorias da motivação que foram surgindo ao longo do tempo e, em especial, a apresentação de um estudo empírico. $O$ objetivo principal desta investigação é verificar a aplicabilidade da Teoria dos Dois Fatores de Herzberg na sociedade portuguesa atual e mostrar a importância da motivação do colaborador para o desempenho organizacional, contribuindo para a divulgação da importância desta temática na investigação científica. Quanto ao estudo empírico foi desenvolvida uma investigação de cariz quantitativo, descritivo e correlacional. A recolha de dados foi feita através de um inquérito por questionário online, tendo sido constituída uma amostra não probabilística de 45 indivíduos e, para o tratamento dos dados obtidos, foi utilizado o programa estatístico SPSS 23. Neste estudo empírico foi possível verificar a aplicabilidade da Teoria dos Dois Fatores na sociedade portuguesa, uma vez que se verifica uma relação direta entre os fatores higiénicos ou motivacionais com a motivação do colaborador, a definição da missão, o desenvolvimento pessoal e profissional e a satisfação do colaborador, apesar de não se verificar a mesma relação relativa às atividades de acolhimento/formação e a partilha de resultados.
\end{abstract}

Palavras-chave: Motivação, Teorias da Motivação e Teoria dos Dois Fatores.

\footnotetext{
${ }^{1}$ Mestrando em Gestão, Especialização em Gestão de Negócios na Universidade Católica Portuguesa - Viseu. E-mail: ferreiraribeiro.marco@gmail.com

2 Prof. Auxiliar - Departamento de Economia, Gestão e Ciências Sociais da Universidade Católica Portuguesa. E-mail: clotilde.passos@gmail.com

3 Prof. Auxiliar - Departamento de Economia, Gestão e Ciências Sociais da Universidade Católica Portuguesa. E-mail: ppereira@viseu.ucp.pt
} 


\begin{abstract}
This research aims to address the theme of organizational motivation, the historical evolution of the perception of motivation on the part of management, their relationship with job satisfaction, the different theories of motivation that have emerged over time and the presentation of an empirical investigation. The objective of this investigation is to verify the applicability of Herzberg's Theory of Two Factors in the current Portuguese society and show the importance of employee motivation for organizational performance, ccontributing to the dissemination of the importance of this thematic in scientific research. As for the empirical study, a quantitative, descriptive, and correlative research was developed. The data collection was done through an online questionnaire survey, and a sample of 45 individuals was obtained and the SPSS 23 statistical software was used to treat the data obtained.in this empirical study it was possible to verify the applicability of the Theory of two factors in Portuguese society, since there is a direct relationship between the hygienic or motivational factors with the motivation of the employee, the definition of the mission, personal and professional development and satisfaction of the employee, although the same relation regarding the activities of reception / formation and the sharing of results is not verified.
\end{abstract}

Keywords: Motivation, Theories of Motivation and Theory of Two Factors.

\title{
INTRODUÇÃO
}

A motivação (derivada do latim movere) é entendida como uma tensão afetiva suscetível de desencadear uma atividade com vista a alcançar um determinado desejo/objetivo (Eccheli, 2008), que pode surgir de uma necessidade, isto é, um estado interno ao indivíduo, capaz de induzir à ação, com vista a alcançar resultados atrativos que visam a satisfação de uma necessidade (Oliveira, 2010). Neste sentido, a motivação é uma força inata que nos impulsiona, tornando-nos capazes de alcançar os nossos objetivos. A motivação é baseada em emoções, em especial, no desejo por experiências emocionais positivas e, consequentemente, por evitar experiências negativas, onde a definição de positivo ou negativo varia de individuo para individuo e do seu estado psicológico no momento, independentemente de normas sociais.

Para alcançar o potencial máximo dos recursos humanos de modo a maximizar o seu desempenho é essencial que os indivíduos se sintam motivados. A motivação dos colaboradores tem vindo a assumir uma importância crescente para as organizações. É através de colaboradores 
motivados que as organizações conseguem obter altos níveis de desempenho e produtividade, através dos esforços coletivo destes, que constituem a energia motriz para o alcance das metas organizacionais e dos resultados desejados (Nascimento, 2008). Neste sentido, a motivação dos colaboradores é essencial ao bom desempenho das organizações e, consequentemente, à sua sobrevivência. Colaboradores desmotivados não permitem que as organizações se desenvolvam e cresçam de forma sustentável.

A motivação surge como uma forma de explicar o modo como os indivíduos agem numa organização. Ao longo do tempo foram surgindo várias teorias sobre motivação, no entanto, perceber como os indivíduos são motivados é um processo complexo (Fraga, 2005). A compreensão dos motivos subjacentes ao comportamento humano tem vindo ao longo dos tempos a ser tema de investigação para muitos investigadores.

Esta investigação tem por interesse aprofundar a temática da motivação organizacional, em especial as diferentes teorias que explicam o processo de motivação. Este estudo visa abordar o conceito de motivação, a evolução histórica da sua perceção por parte da gestão, as diferentes teorias da motivação que foram surgindo ao longo do tempo e, em especial, a apresentação de um estudo empírico, que tem como objetivo verificar se a Teoria dos Dois Fatores de Herzberg se adequa à realidade portuguesa.

\section{EVOLUÇÃO DA MOTIVAÇÃO ORGANIZACIONAL}

De acordo com os diversos modelos de gestão que vigoraram, desde o Taylorismo até aos modelos contemporâneos atuais, o conceito de motivação foi-se desenvolvendo e evoluindo ao longo do tempo. De acordo com o modelo de gestão vigente os fatores considerados como motivadores foram-se alterando. Com a publicação, em 1911, d'Os Princípios da Administração Científica, por Frederick Taylor, foram estabelecidos como prioridade para a gestão de uma organização a eficiência e eficácia. Em 1916, Henry Fayol, crítico de Taylor, publicou Administration Industrielle et Générale e desenvolveu a Teoria da Anatomia e Fisiologia da Organização. Ao contrário de Taylor, Fayol analisou a estrutura hierárquica da organização de baixo para cima e percebeu que cada trabalhador recebia ordens de vários superiores diferentes, sendo esta a sua principal crítica. Por outro lado, Max Weber desenvolveu um modelo burocrático de gestão assente na formalidade, 
racionalidade e previsibilidade total de funcionamento. Em ambas as três abordagens clássicas, de acordo com Fraga (2005), o trabalhador era motivado à base de recompensas pecuniárias e punições.

Esta abordagem de motivação foi alterada com o surgimento de uma nova abordagem de administração, a abordagem das relações humanas por Elton Mayo. $\mathrm{O}$ foco no trabalhador como homo economics (homem máquina) passou a ser visto como homo social, isto é, ao contrário das abordagens clássica, esta nova abordagem tem como foco a ênfase nas pessoas. De acordo com Tadin, Rodrigues, Dalsoquio, Guabiraba e Miranda (2006), as três principais características desses modelos são: o ser humano não pode ser reduzido a um ser cujo comportamento é simples e mecânico; o homem é, ao mesmo tempo, guiado pelo sistema social e pelas hierarquias e todos os homens possuem necessidades de segurança, afeto, aprovação social, prestígio e autorrealização. Através desta abordagem foi possível estabelecer uma definição de motivação aceitável atualmente. A motivação é uma tensão afetiva suscetível de desencadear atividade com vista a alcançar um objetivo desejado. Para Maslow (1954), citado por Sampaio (2009, p.6), “O homem é um animal com vários desejos e raramente atinge um estado de completa satisfação exceto por um curto período de tempo. Assim que um desejo é satisfeito, outro surge e assume o seu lugar".

A abordagem sistémica-contingencial é a abordagem atualmente em vigor, que define o sistema organizacional como resultante da interação ambiente-organização, sendo o ambiente um agente participativo na vida organizacional (Tureta, Rosa \& Ávila, 2006).

\section{MOTIVAÇÃO E SATISFAÇÃO NO TRABALHO}

A satisfação no trabalho diz respeito a uma resposta emocional em relação a uma situação do trabalho, estando relacionado, em muitos casos, com o exceder da expetativa, ou seja, trata-se de um estado emocional positivo resultante de uma avaliação afetiva/cognitiva de uma vivência no local de trabalho (Cortinhas, 2014).

Lima, Vala e Monteiro (1988) definem três níveis de satisfação organizacional. O primeiro nível prende-se com variáveis situacionais utilizadas na explicação da satisfação, tais como as caraterísticas da função, do processo de tomada de decisão e do reforço. O segundo nível de satisfação compreende as variáveis individuais como a discrepância entre expetativas e resposta da organização e o terceiro nível com a 
interação social que compreende as variáveis de comparação social, processamento da informação e cultura organizacional.

Existem várias teorias que descrevem o processo de satisfação (Cidade, 2013), tais como a teoria da realização - no caso das necessidades individuais do colaborador estarem satisfeitas, este estará satisfeito com o seu trabalho; a teoria da discrepância - a satisfação ocorre quando a recompensa efetiva é igual à recompensa desejada; teoria da equidade de Adams - essencialmente tida como uma teoria motivacional pode ser considerada também uma teoria de satisfação na medida em que a equidade gera satisfação no trabalho; teoria das facetas - a satisfação está dependente das diversas facetas, tais como as recompensas monetárias e a satisfação com a supervisão, por exemplo, teoria da comparação social - quanto menor a diferença entre o que o indivíduo deseja e aquilo que realmente deseja, maior a satisfação (Cidade, 2013).

Tanto a motivação como a satisfação dos colaboradores são muito importantes para o desenvolvimento e crescimento sustentável da organização (Bergamini \& Bullet, 1990). Relacionando estes dois conceitos podemos obter quatro estados: I - Estar motivado e satisfeito; II - Estar satisfeito sem estar motivado; III - Estar motivado sem estar satisfeito; IV - Estar desmotivado e insatisfeito (Cortinhas, 2014).

Neste sentido, a motivação e a satisfação são dois conceitos que se complementam e influenciam diversas variáveis organizacionais, como, por exemplo, a eficiência, a eficácia e a produtividade. Neste sentido, é importante ter em atenção estes dois conceitos na gestão do desempenho organizacional em relação ao capital humano (Figueiredo, 2012).

\section{TEORIAS DA MOTIVAÇÃO}

A motivação do colaborador tem vindo a ser tema de diversos estudos científicos e desenvolvimento de várias teorias que visam explicar quais os fatores que estão na origem da motivação. A validade científica de uma teoria não anula as demais, pelo contrário, de acordo com Boas, Esteves e Ferreira (2006), muitas teorias, na verdade, são complementares e o desafio consiste em perceber o seu inter-relacionamento.

De modo geral, todas as teorias apresentadas defendem o contributo positivo da motivação do colaborador para a organização, pelo que é unanime manter o colaborador motivado como forma de garantir um bom desempenho empresarial. 


\subsection{Teorias de conteúdo}

\subsubsection{Teoria da Hierarquia das Necessidades}

Uma das teorias mais conhecidas é a Teoria da Hierarquia das Necessidades de Maslow. Tendo como base o estudo de outros investigadores como Reich, Jung Adler, Fromm e Freud e várias investigações empíricas por si realizadas, Maslow desenvolveu, em 1954, uma teoria que identifica as principais necessidades do ser humano e as classifica numa escala ascendente de hierarquização. Assim, à medida que as necessidades do nível hierárquico inferior vão sendo satisfeitas, surgem como seguintes as necessidades da categoria imediatamente superior que passam a motivar mais intensamente o comportamento (Bueno, 2002). A escala proposta por Maslow é constituída por cinco categorias:

1. Necessidades fisiológicas: visam manter o bom funcionamento do organismo, sendo indispensável à sobrevivência.

2. Necessidades de segurança: sentimento de proteção contra eventuais perigos, sejam estes acidentes, doenças, catástrofes, instabilidade económica, entre outros.

3. Necessidades sociais: desejo de pertença e participação, afeto e amizade.

4. Necessidades de estima: desejo de destacar-se no grupo que integra e de ser prestigiado pelos demais.

5. Necessidades de autorrealização: desejo de crescimento pessoal e profissional, ou seja, de aprimoramento das capacidades pessoais e de excelência, constituindo um desafio permanente para o indivíduo.

De acordo com Pérez-Ramos (1990), as contribuições de Maslow têm servido como base para o desenvolvimento de novos modelos teóricos e, por outro lado, tem possibilitado a organização de programas de desenvolvimento de recursos humanos assente na perceção da categorização destas necessidades.

\subsubsection{Teoria dos Dois Fatores}

Outra importante teoria da motivação é a Teoria dos Dois Fatores concebida por Herzberg em 1959. A tentativa deste investigador em perceber a razão das necessidades, motivos e atitudes dos indivíduos no local de trabalho permitiu formular as suposições fundamentais para compreender o comportamento dos colaboradores através de dois tipos de 
fatores. De acordo com Herzberg, Mausner e Snyderman (1959), os fatores higiénicos não motivam por si só, mas não estando presentes provocam insatisfação/desmotivação. São exemplos a política e administração/gestão da empresa, a supervisão, o salário, as relações humanas e as condições de trabalho. Além destes fatores, devem-se considerar os fatores motivacionais, aqueles que realmente motivam. São exemplos de fatores motivacionais a realização, o reconhecimento, o trabalho em si, a responsabilidade e o progresso/desenvolvimento.

Estes dois grupos de fatores são independentes (Andrade, Pereira \& Ckagnazaroff, 2007). Os fatores motivacionais são supostos causadores de satisfação, quando estão presentes, embora não sejam causadores de insatisfação quando estão ausentes. Da mesma forma, a presença dos fatores higiénicos não geram motivação, mas a sua ausência gera insatisfação.

Como Pérez-Ramos (1990) e Gawel (1997) referem, podemos, assim, perceber que as proposições de Herzberg são compatíveis com as de Maslow, embora apresentem diferentes pontos de vista. Neste sentido, os fatores de higiene de Herzberg relacionam-se diretamente com as necessidades fisiológicas, de segurança e sociais de Maslow, enquanto que os fatores de motivação descritos por Herzberg correspondem às necessidades de estima e de autorrealização do esquema de Maslow.

\subsubsection{Teoria $X$ e $Y$}

Douglas McGregor propôs, na década de 1960, duas visões distintas de gestão de organizações: por um lado, uma teoria tradicional e mecanicista, designada de Teoria X; e por outro, uma teoria baseada nas conceções modernas a respeito do comportamento humano, designada de Teoria Y. Assim, enquanto a Teoria X está relacionada com as abordagens clássicas de administração, a Teoria Y está relacionada com a abordagem das relações humanas ou a abordagem sistémicacontingencial.

Neste sentido, de acordo com Cunha, Rego, Cunha e Cardoso (2007), a Teoria $X$ baseia-se na convicção de que o homem é indolente e preguiçoso por natureza, sem ambição, que privilegia os seus interesses pessoais e resiste à mudança, uma vez que ambiciona segurança e não correr riscos, sendo que, a esta teoria está associada um estilo de liderança autocrático. Segundo Galhanas (2009), os trabalhadores devem ser recompensados, punidos, coagidos e controlados através de incentivos 
económicos, principal fonte de motivação do colaborador. A organização utiliza a remuneração como um meio de recompensa ou punição.

De acordo com Galhanas (2009), a Teoria Y apresenta um estilo mais moderno e baseia-se na convicção de que o trabalho pode ser uma fonte de satisfação para o colaborador. Nestes casos, a aplicação do esforço físico ou mental no decorrer do trabalho é tão natural quanto se divertir ou descansar, pelo que o colaborador não é, por natureza, passivo ou resistente às necessidades da empresa. Nos casos de fuga à responsabilidade, falta de ambição e passividade estes são considerados como consequências da experiência insatisfatória de cada um e não uma característica humana. Neste sentido, de acordo com Cunha et al (2007), a motivação, o potencial de desenvolvimento, a capacidade de assumir responsabilidades e de dirigir o comportamento para os objetivos da empresa são fatores presentes nas pessoas. Cabe, assim, à gestão proporcionar condições para que as pessoas reconheçam e desenvolvam, por si mesmas, essas características.

\subsubsection{Teoria ERG (Existence - Relatedness - Growth)}

A teoria ERG (Existência - Relacionamento - Crescimento) foi formulada em 1969 por Alderfer e deriva, tal como a anterior da Teoria da Hierarquia das Necessidades de Maslow, diferindo desta, não apenas no número de categorias da hierarquia (três em Alderfer e cinco em Maslow), mas também no sentido/direção do desenvolvimento motivacional. Enquanto que para Maslow existe apenas um único sentido ascendente (direção de satisfação-progressão), para Alderfer o sentido inverso, descendente, também será válido (direção frustração-regressão) (Ferreira, Boas, Esteves, Fuerth \& Silva, 2006).

\subsubsection{Teoria do Work Design}

A Teoria do Work Design, também conhecida como Modelo das Caraterísticas do Trabalho, foi desenvolvida por J. Richard Hackman e Greg Oldham em 1976. Esta teoria determina que a variedade de funções, identidade, significado das tarefas, autonomia e feedback são determinantes da motivação do colaborador e resultará em um desempenho excedente e mais satisfatório (Pedroso, Pilatti, Santos \& Junior, 2010).

Devemos, assim, de acordo com Pedroso e Pilatti (2010), considerar a seguinte fórmula do potencial motivador (PM): 


$$
\mathrm{PM}=\frac{\mathrm{VF}+\mathrm{I}+\mathrm{ST}}{3} \times \mathrm{A} \times \mathrm{F}
$$

PM: Potencial Motivador; VF: Variedade de Funções; I: Identidade; ST: Significado das Tarefas; A: Autonomia; F: Feedback

Uma baixa em autonomia ou feedback irá comprometer substancialmente o potencial motivador, pois estes são indispensáveis para motivar o colaborador. Ou seja, à medida que a responsabilidade, liberdade e reconhecimento dos resultados do funcionário aumenta, este torna-se cada vez mais estimulado. Será necessário obter o mínimo de classificação nestes dois fatores, pois caso um deles seja zero, não existe potencial motivador. Pelo contrário, uma nota baixa em uma das três características do trabalho, não necessariamente acabará por reduzir o potencial motivador, porque uma forte presença de um dos três atributos pode compensar a ausência dos outros.

\subsection{Teorias de processo}

\subsubsection{Teoria da Dissonância Cognitiva}

Formulada por Festinger em 1954, a Teoria da Dissonância Cognitiva parte do pressuposto de que o ser humano avalia sua própria autoimagem, utilizando-a como termo de comparação com indivíduos com caraterísticas semelhantes às suas. Com a perceção de incongruências ou discordâncias na avaliação entre a perceção de si mesmo e do outro, surge um fenómeno psicológico conhecido como dissonância cognitiva (Spink, 2007). Este fenómeno leva o indivíduo a tentar superar o outro de forma a estabelecer a consonância original entendida como um equilíbrio psicológico. Esta tentativa de recuperar o equilíbrio pode originar um comportamento errático ou irracional contribuindo para um desequilíbrio maior (Gawel, 1997).

Um exemplo do ponto de vista organizacional reside no fato de que se a cultura da empresa não representa os valores e crenças do indivíduo ocorre a dissonância e o relacionamento entre as partes pode ser prejudicado se esta não for eliminada. Sendo assim, a escolha de uma organização para se trabalhar vai muito além de aspetos financeiros. $\mathrm{O}$ ideal é que seja feita uma análise sobre quais são os valores da empresa para verificar se são consonantes com os do indivíduo que pretende fazer 
parte daquela organização de modo a evitar possíveis dissonâncias cognitivas.

\subsubsection{Teoria da Equidade}

A Teoria da Dissonância Cognitiva tem vindo a sofrer alterações. Em 1961, Stacy Adams alterou alguns dos seus aspetos instrumentais e criou a Teoria da Equidade. A necessidade de justiça no local de trabalho levou à criação desta teoria. Devemos considerar, à luz desta teoria, que os colaboradores analisam o que possuem da organização, tais como salários, benefícios, promoções, entre outros e as suas competências, tais como experiência, escolaridade e empenho e fazem comparação com colaboradores que desempenhem as mesmas funções (Lobos, 1975). De acordo com Lima, Vala e Monteiro (1988), o objetivo será averiguar a existência de equidade entre os seus benefícios com os dos demais. No caso dos seus benefícios serem subcompensados, esta tensão cria um sentimento de injustiça; caso o indivíduo se veja com excesso de recompensas, esta tensão surge no indivíduo como um sentimento de culpa. Para além disso, devemos ainda salientar que esta comparação resulta apenas da perceção do colaborador e não de uma medição objetiva (Lobos, 1975).

\subsubsection{Teoria das Expetativas}

A Teoria das Expetativas foi idealizada por Victor Vroom em 1964 e, segundo Boas (2006), tem como principal expoente, a motivação ser desenvolvida de acordo as perceções do indivíduo acerca da relação entre os esforços que entende que devam ser realizados para alcançar um determinado objetivo ou meta e o valor que lhes atribui, daí que o comportamento escolhido é, em regra, aquele que conduz a maiores ganhos para a pessoa. Assim, a motivação será uma função da valência (força de atração/repulsa percebida), da instrumentalidade (relação percetível entre os resultados desejados e a potencialidade de desempenho) e da expectativa (representação antecipada da decisão que deve ser tomada) (Lobos, 1975). Estes três fatores devem ser combinados por multiplicação, uma vez que na ausência de um, o nível motivacional também será nulo. Por outro lado, quanto maior a intensidade desses fatores, maior será o nível motivacional resultante. De acordo com Caldas e Filho (2008), esta relação pode ser representada pela seguinte equação:

Motivaçâo $=f($ valência $\times$ instrumentalidade $\times$ expectativa $)$ 


\subsubsection{Outras teorias}

Para além das teorias anteriormente apresentadas podemos ainda considerar a existência de outras. Porter e Lawler formulam em 1968 a Teoria do Desempenho-Ação que defende que o colaborador pode antecipar/prever eventos e propor a remuneração/gratificação desejada e a satisfação das suas necessidades. Em 1990, surge a Teoria dos Objetivos, por Locke e Lather, em que a motivação resulta da comparação que é feita entre os objetivos propostos e o desempenho. Em 1971, surge a Teoria da Atribuição Causal segundo a qual os possíveis efeitos derivados de um determinado fator causal, podem ser atenuados ou modificados pela influência de outros incidentes. Em 1972, Bem formula a Teoria da Auto-perceção em que o colaborador tende a trabalhar a sua perceção de maneira a tornar-se semelhante aos restantes, sendo a promoção da semelhança um fator de motivação. Em 1975, Ryan e Decin formulam a Teoria da Motivação Intrínseca e do Envolvimento que determina que as emoções de prazer são determinantes de um comportamento motivado. No mesmo ano, Cabe e Locke formulam a Teoria do Estabelecimento de Metas que define o estabelecimento de metas, vistas como um objetivo desejado, o principal fator motivador do colaborador. Ryan e Decin, juntamente com DeCharmes formulam, em 1985, a Teoria da Avaliação Cognitiva que destaca a importância da interação dos fatores de caráter individual (como o colaborador) e os de natureza ambiental (organização) para explicar o fenômeno e os processos motivacionais

\section{PRIMAZIA DA TEORIA DOS DOIS FATORES NESTA INVESTIGAÇÃO}

Neste estudo, a investigação empírica visa averiguar se a Teoria dos Dois Fatores de Herzberg se adequa à realidade portuguesa. A escolha desta teoria deve-se ao fato de tentar descrever os fatores que geram insatisfação (ausência de fatores higiénicos e motivacionais), aqueles que seriam responsáveis pela satisfação (presença dos fatores higiénicos) e ainda os responsáveis pela motivação (presença de fatores higiénicos e motivacionais). Neste sentido, o objetivo foi perceber se estes fatores se adequam à realidade portuguesa. A escolha desta teoria, para investigação empírica, em deterioramento das restantes, deve-se essencialmente ao fato de se considerar que seja a mais complexa. Contudo todas as outras teorias são válidas na explicação do processo de motivação, pelo que 
seria interessante do ponto de vista científico a realização de uma investigação semelhante para as restantes teorias.

\section{INVESTIGAÇÃO EMPÍRICA}

\subsection{Metodologia}

A investigação efetuada tem como principal objetivo avaliar se a Teoria dos Dois Fatores de Herzberg se adequa à realidade portuguesa.

Neste sentido, foi desenvolvida uma investigação de cariz quantitativo, descritivo e correlacional. A recolha de dados foi feita através de um inquérito por questionário online, construído através da adaptação de Júnior e Oliveira (2009), tendo sido aplicado durante os meses de novembro e dezembro de 2017 e constituída uma amostra não probabilística de 45 indivíduos, através da utilização de um processo conhecido como "bola de neve".

Considerando a Teoria Dos Fatores de Herzberg, que perceciona a existência de dois fatores, os fatores higiénicos e os fatores motivacionais, os itens foram classificados de acordo com a investigação teórica realizada anteriormente. Neste sentido, os itens 1 . Sinto-me seguro contra eventuais despedimentos, 2. Os regulamentos e políticas organizacionais são justos, 3. Tenho boas condições de trabalho, 4. O meu trabalho proporciona-me prestígio, 5. Tenho uma vida pessoal satisfatória, 6. Tenho um bom relacionamento com os meus superiores, 7 . A função que desempenho está de acordo com o trabalho que realizo e 8 . Tenho um salário adequado às minhas funções são considerados como fatores higiénicos, enquanto que os restantes itens, 9. São criadas oportunidades de crescimento pessoal, 10. Tenho autonomia e responsabilidade no meu trabalho, 11 . O meu trabalho proporciona-me oportunidades de progresso na carreira, 12. Tenho o devido reconhecimento pelo trabalho que realizo e 13. Tenho um trabalho desafiador e interessante são considerados como fatores motivacionais.

Para o tratamento dos dados obtidos, foi utilizado o programa estatístico SPSS 23.0, sendo que, ao longo da aplicação dos testes estatísticos foi utilizado um valor de referência de 5\%. Os testes estatísticos utilizados foram o teste paramétrico $t$ de Student, por forma a verificar a significância das diferenças entre os valores médios observadas para ambos os grupos da variável nominal dicotómica, como explicado por Marôco (2011, p. 199-204), tendo sido feita a análise do pressuposto da normalidade que permite escolher entre a utilização de testes paramétricos ou não paramétricos, de acordo com Marôco (2011, p. 
185-195), com o teste de Kolmogorov-Smirnov, tendo-se verificado que as variáveis em estudo seguem a distribuição normal.

Neste sentido, foram formuladas as seguintes hipóteses de investigação:

$\mathrm{H}_{1}$ : Os fatores higiénicos relacionam-se positivamente com a motivação do colaborador.

$\mathrm{H}_{2}$ : Os fatores motivacionais relacionam-se positivamente com a motivação do colaborador.

$\mathrm{H}_{3}$ : Os fatores higiénicos relacionam-se positivamente com a existência de atividades de acolhimento/formação.

$\mathrm{H}_{4}$ : Os fatores motivacionais relacionam-se positivamente com a existência de atividades de acolhimento/formação.

$\mathrm{H}_{5}$ : Os fatores higiénicos relacionam-se positivamente com a definição da missão.

$\mathrm{H}_{6}$ : Os fatores motivacionais relacionam-se positivamente com a definição da missão.

$\mathrm{H}_{7}$ : Os fatores higiénicos relacionam-se positivamente com o desenvolvimento pessoal e profissional.

$\mathrm{H}_{8}$ : Os fatores motivacionais relacionam-se positivamente com o desenvolvimento pessoal e profissional.

$\mathrm{H}_{9}$ : Os fatores higiénicos relacionam-se positivamente com a satisfação do colaborador.

$\mathrm{H}_{10}$ : Os fatores motivacionais relacionam-se positivamente com a satisfação do colaborador.

$\mathrm{H}_{11}$ : Os fatores higiénicos relacionam-se positivamente com a partilha de resultados.

$\mathrm{H}_{12}$ : Os fatores motivacionais relacionam-se positivamente com a partilha de resultados.

\subsection{Descrição da amostra}

Dos inquiridos, $20(44,4 \%)$ são do sexo feminino e 25 (55,6\%)do sexo masculino. A totalidade dos inquiridos reside em Portugal, mais propriamente nas cidades de Viseu, Funchal, Lisboa, Oeiras, Vila Nova de Gaia, Coimbra, Seia, Lamego, Porto, Covilhã e Tavira. Um elemento da amostra (2,2\%) tinha uma idade compreendida entre 18 a 20 anos, 10 $(22,2 \%)$ entre 21 a $30,17(37,8 \%)$ entre 31 a $40,11(24,4 \%)$ entre 41 a 50 , cinco $(11,1 \%)$ entre 51 a 60 , e um indivíduo $(2,2 \%)$ entre 61 a 70 anos. Relativamente às habilitações, um inquirido $(2,2 \%)$ tinha uma escolaridade até ao $4^{\circ}$ ano, também um $(2,2 \%)$ tinha do $5^{\circ}$ ao $9^{\circ}$ ano, 14 
inquiridos $(31,1 \%)$ tinham $10^{\circ}$ ao $12^{\circ}$ ano, $18(40 \%)$ tinham licenciatura e 11 inquiridos $(24,4 \%)$ uma pós-graduação. Quanto ao estado civil, 23 inquiridos $(51,1)$ são solteiros, $14(31,1)$ casados ou em união de factos e $8(17,8 \%)$ divorciados/separados. Dos inquiridos, $22(48,9 \%)$ tem filhos, enquanto que os restantes $23(51,1 \%)$ não têm filhos. Quanto à situação face ao emprego, 40 inquiridos $(88,9 \%)$ referiram estar empregados e 5 $(11,1 \%)$ indicaram estar desempregados. Relativamente ao setor de atividade, 2 inquiridos $(4,4 \%)$ classificaram o seu setor de atividade como a saúde, $6(13,3 \%)$ a educação, $4(8,9 \%)$ a função pública, $3(6,7 \%)$ o desporto, $23(51,1 \%)$ os serviços, um $(2,2 \%)$ a construção e $6(13,3 \%)$ a indústria. Dois indivíduos $(4,4 \%)$ estão empregados há menos de um ano, $15(33,3 \%)$ entre 1 a 3 anos, $6(13,3)$ entre 4 a 6 anos e 17 (37,8\%) estão empregados há mais de 6 anos. Quanto à implementação geográfica da organização em que trabalham, 24 inquiridos $(53,3 \%)$ referiram ser regional, $20(44,4 \%)$ ser nacional e um indivíduo $(2,2 \%)$ multinacional.

\subsection{Resultados}

Quanto à questão "Quando um novo colaborador entra na empresa é feito um acolhimento ou formação estruturados com objetivos específicos?", 27 inquiridos (60\%) respondeu que na sua organização são realizadas atividades de acolhimento e formação, enquanto que 18 inquiridos (40\%) refere negativamente. Quanto à questão referente à existência de uma missão bem definida, 30 inquiridos $(66,7 \%)$ referem que a sua organização possui uma missão bem definida, enquanto que 7 inquiridos $(15,6 \%)$ referem que a sua organização não possui uma missão definida. Relativamente à questão da preocupação, por parte da organização, com o desenvolvimento pessoal e profissional dos colaboradores, 22 inquiridos $(48,9 \%)$ refere que efetivamente existe uma preocupação constante com o seu desenvolvimento pessoal e profissional, enquanto que 18 inquiridos (40\%) considera o oposto. Quanto à questão relativa à preocupação, por parte da organização, com a satisfação dos colaboradores, 14 inquiridos $(31,1 \%)$ considera que efetivamente existe esse tipo de preocupação, enquanto que 25 inquiridos $(55,6 \%)$ refere o oposto. Quanto à questão referente à partilha de resultados, 5 inquiridos referem que a sua organização partilha os resultados com os colaboradores, enquanto que 33 inquiridos $(73,3 \%)$ refere que não partilha. Relativamente à questão "Sinto-me motivado no local de trabalho?", 29 dos inquiridos $(64,4 \%)$ responderam que se sentem motivados, enquanto que os restantes 16 inquiridos $(35,6 \%)$ refere o oposto. 
A motivação do colaborador foi também avaliada através da utilização de 13 itens e uma escala de Likert de quatro pontos distribuídos de 1, Discordo Totalmente, a 5, Concordo Totalmente, tendo-se verificados os resultados apresentados na tabela seguinte.

\section{Tabela 1}

Estatísticas: Itens da Escala de Motivação

\begin{tabular}{lcccc}
\hline & & & $\begin{array}{c}\text { Desvio } \\
\text { Padrão }\end{array}$ & $\begin{array}{c}\text { Coef. } \\
\text { Variação }\end{array}$ \\
\hline 1. Sinto-me seguro contra eventuais despedimentos & 45 & 2,44 & 0,81 & $33 \%$ \\
2. Os regulamentos e políticas organizacionais são justos & 45 & 2,49 & 0,87 & $35 \%$ \\
3. Tenho boas condições de trabalho & 45 & 2,89 & 0,88 & $31 \%$ \\
4. O meu trabalho proporciona-me prestígio & 45 & 2,64 & 1,03 & $39 \%$ \\
5. Tenho uma vida pessoal satisfatória & 45 & 3,04 & 0,85 & $28 \%$ \\
6. Tenho um bom relacionamento com os meus superiores & 45 & 2,82 & 0,91 & $32 \%$ \\
7. A função que desempenho está de acordo com o trabalho & 45 & 2,89 & 0,96 & $33 \%$ \\
que realizo & & & & \\
8. Tenho um salário adequado às minhas funções & 45 & 2,44 & 1,06 & $43 \%$ \\
9. São criadas oportunidades de crescimento pessoal & 45 & 2,38 & 0,98 & $41 \%$ \\
10. Tenho autonomia e responsabilidade no meu trabalho & 45 & 2,87 & 0,97 & $34 \%$ \\
11. O meu trabalho proporciona-me oportunidades & 45 & 2,56 & 1,03 & $40 \%$ \\
progresso na carreira & & & & \\
12. Tenho o devido reconhecimento pelo trabalho que realizo & 45 & 2,56 & 1,03 & $40 \%$ \\
13. Tenho um trabalho desafiador e interessante & 45 & 2,62 & 1,13 & $43 \%$ \\
\hline
\end{tabular}

Os valores indicados reportam-se à escala de medida:

1-Discordo totalmente; 2-Discordo; 3-Concordo; 4-Concordo totalmente.

Em média, a concordância é superior para " 5 . Tenho uma vida pessoal satisfatória" (M=3,04), seguido de "3. Tenho boas condições de trabalho" $(M=2,89)$, "7. A função que desempenho está de acordo com o trabalho que realizo" (M=2,89) e "9. Tenho autonomia e responsabilidade no meu trabalho" $(M=2,87)$ e " 6 . Tenho um bom relacionamento com os meus superiores" $(\mathrm{M}=2,82)$, depois de "4. O meu trabalho proporciona-me prestígio" (M=2,64) e "13. Tenho um trabalho desafiador e interessante" $(\mathrm{M}=2,62)$ e ainda de "10. O meu trabalho proporciona-me oportunidades de progresso na carreira" $(\mathrm{M}=2,56)$ e "12. Tenho o devido reconhecimento pelo trabalho que realizo" $(\mathrm{M}=2,56)$, todos com valor médio superior ao ponto intermédio da escala; depois surge "2. Os regulamentos e políticas organizacionais são justos" $(M=2,49)$, seguido de "1. Sinto-me seguro contra eventuais despedimentos" $(\mathrm{M}=2,44)$ e "11. Tenho um salário adequado às minhas funções" $(M=2,44)$ e finalmente de "8. São criadas oportunidades de crescimento pessoal" $(M=2,38)$, tendo estes itens valor médio inferior ao ponto intermédio da escala. 
Começando por analisar os fatores higiénicos, foi realizada uma análise de confiabilidade através da utilização do Alfa de Cronbach, isto é, uma análise à consistência interna/fiabilidade dos diferentes itens que constituem os fatores higiénicos. Neste sentido, o fator em causa é constituído pelos 8 itens referidos anteriormente e possui um Alfa de Cronbach de 0,914, o que significa a existência de um nível bom de fiabilidade.

Tabela 2

Alfa de Cronbach no caso de exclusão individual do item para os Fatores Higiénicos

\begin{tabular}{lrc}
\hline & $\begin{array}{c}\text { Correlação de } \\
\text { item total } \\
\text { corrigida }\end{array}$ & $\begin{array}{c}\text { Alfa de Cronbach } \\
\text { se o item for } \\
\text { excluído }\end{array}$ \\
\hline 1. Sinto-me seguro contra eventuais despedimentos &, 671 &, 907 \\
2. Os regulamentos e políticas organizacionais são justos &, 704 &, 905 \\
3. Tenho boas condições de trabalho &, 803 &, 897 \\
4. O meu trabalho proporciona-me prestígio &, 774 &, 899 \\
5. Tenho uma vida pessoal satisfatória &, 659 &, 908 \\
6. Tenho um bom relacionamento com os meus superiores &, 751 &, 901 \\
7. A função que desempenho está de acordo com o trabalho &, 690 &, 906 \\
que realizo &, 719 &, 904 \\
8. Tenho um salário adequado às minhas funções & & \\
\hline
\end{tabular}

Efetuando a mesma análise para os fatores motivacionais verifica-se que este é constituído por 5 itens e por um Alfa de Cronbach de 0,917. Neste sentido, o nível de fiabilidade é considerado bom.

Tabela 3

Alfa de Cronbach no caso de exclusão individual do item para os Fatores Motivacionais

\begin{tabular}{lcc}
\hline & $\begin{array}{c}\text { Correlação } \\
\text { de item total } \\
\text { corrigida }\end{array}$ & $\begin{array}{c}\text { Alfa de Cronbach } \\
\text { se o item for } \\
\text { excluído }\end{array}$ \\
\hline 9. São criadas oportunidades de crescimento pessoal &, 804 &, 896 \\
10. Tenho autonomia e responsabilidade no meu trabalho &, 723 &, 911 \\
11. O meu trabalho proporciona-me oportunidades de progresso &, 832 &, 889 \\
na carreira &, 713 &, 913 \\
12. Tenho o devido reconhecimento pelo trabalho que realizo &, 871 &, 881 \\
13. Tenho um trabalho desafiador e interessante & \\
\hline
\end{tabular}

Verifica-se para ambas as dimensões que ao avaliar a influencia do valor de Alfa de Cronbach, no caso de eliminação de um item individualmente, o Alfa de Cronbach passaria a ser inferior, pelo que nenhum item deve ser eliminado. 


\section{Tabela 4}

Relação entre os fatores (higiénicos e motivacionais) e a motivação: estatísticas descritivas e resultados do teste $\mathrm{t}$

\begin{tabular}{lllllll}
\hline & $\begin{array}{l}\text { Sinto-me } \\
\text { motivado }\end{array}$ & N & Média & $\begin{array}{l}\text { Desvio } \\
\text { Padrão }\end{array}$ & Teste t & $\mathrm{p}$ \\
\hline Fatores higiénicos & Sim & 29 & 3,13 & 0,436 & $\mathrm{t} 43=8,061$ & $* * * 0,000$ \\
& Não & 16 & 1,95 & 0,521 & & \\
Fatores motivacionais & Sim & 29 & 3,14 & 0,532 & $\mathrm{t}_{43}=9,578$ & $* * * 0,000$ \\
& Não & 16 & 1,61 & 0,470 & & \\
\hline
\end{tabular}

$* * *$ diferença significativa para $\mathrm{p}<0,001$

Para avaliar a relação entre os fatores (higiénicos e motivacionais) e a motivação é utilizado o teste paramétrico $t$ de Student. No entanto, este teste é antecedido pelo teste de Levene, isto é, um teste de hipótese à igualdade de variâncias com o teste de Levene, tendo-se verificado a existência de homocedasticidade $(\mathrm{p}=0,322$ para os fatores higiénicos e $\mathrm{p}=0,449$ para os fatores motivacionais).

Para os fatores higiénicos, verifica-se que o seu valor médio é superior para os indivíduos motivados $(\mathrm{M}=3,13)$ em comparação com os desmotivados $(\mathrm{M}=1,95)$, sendo as diferenças estatisticamente significativas $\left(\mathrm{t}_{43}=8,061, \mathrm{p}<0,001\right)$. Os mesmos resultados verificaram-se para os fatores motivacionais com valor médio superior para os indivíduos motivados $(\mathrm{M}=3,14)$ em comparação com os desmotivados $(\mathrm{M}=1,61)$, sendo as diferenças também estatisticamente significativas $\left(\mathrm{t}_{43}=9,578, \mathrm{p}<0,001\right)$.

\section{Tabela 5}

Relação entre os fatores (higiénicos e motivacionais) e o processo de acolhimento/formação: estatísticas descritivas e resultados do teste $\mathrm{t}$

\begin{tabular}{lllllll} 
& $\begin{array}{l}\text { Atividades de } \\
\text { acolhimento/formação }\end{array}$ & N & Média & $\begin{array}{l}\text { Desvio } \\
\text { Padrão }\end{array}$ & Teste t & p \\
\hline Fatores & Sim & 27 & 2,84 & 0,722 & $\mathrm{t}_{43}=1,476$ & $* * 0,147$ \\
higiénicos & Não & 18 & 2,51 & 0,721 & & \\
Fatores & Sim & 27 & 2,74 & 0,813 & $\mathrm{t}_{43}=1,345$ & $* * 0,186$ \\
motivacionais & Não & 18 & 2,38 & 0,989 & & \\
\hline
\end{tabular}

Para avaliar a relação entre os fatores (higiénicos e motivacionais) e o processo de acolhimento/formação é utilizado também o teste paramétrico t de Student. Contudo, o respetivo teste é antecedido pelo teste de Levene, 
tendo sido verificado a existência de homocedasticidade ( $\mathrm{p}=0,893$ para os fatores higiénicos e $\mathrm{p}=0,149$ para os fatores motivacionais).

Neste sentido, para os fatores higiénicos, verifica-se que o seu valor médio é superior para a existência de atividades de acolhimento/formação $(\mathrm{M}=2,84)$ em comparação com a não existência $(\mathrm{M}=2,51)$, não sendo as diferenças estatisticamente significativas $\left(\mathrm{t}_{43}=1,476, \mathrm{p}=0,147\right)$. Os mesmos resultados verificaram-se para os fatores motivacionais com valor médio superior para a existência de atividades de acolhimento/formação $(M=2,74)$ em comparação com a não existência $(\mathrm{M}=2,38)$, não sendo as diferenças também estatisticamente significativas $\left(\mathrm{t}_{43}=1,345, \mathrm{p}=0,186\right)$.

Tabela 6

Relação entre os fatores (higiénicos e motivacionais) e a definição da missão: estatísticas descritivas e resultados do teste $\mathrm{t}$

\begin{tabular}{llllllc}
\hline & $\begin{array}{l}\text { Definição } \\
\text { da missão }\end{array}$ & $\mathrm{N}$ & Média & $\begin{array}{l}\text { Desvio } \\
\text { Padrão }\end{array}$ & Teste t & $\mathrm{p}$ \\
\hline Fatores higiénicos & Sim & 30 & 2,80 & 0,725 & $\mathrm{t}_{35}=1,473$ & 0,150 \\
& Não & 7 & 2,36 & 0,712 & & \\
Fatores motivacionais & Sim & 30 & 2,77 & 0,840 & $\mathrm{~T}_{35}=2,445$ & $* 0,020$ \\
& Não & 7 & 1,91 & 0,823 & & \\
\hline
\end{tabular}

*diferença significativa para $\mathrm{p}<0,05$

Para avaliar a relação entre os fatores (higiénicos e motivacionais) e a definição da missão é utilizado o teste paramétrico $t$ de Student. No entanto, o respetivo teste é antecedido pelo teste de Levene, tendo sido verificado a existência de homocedasticidade ( $\mathrm{p}=0,584$ para os fatores higiénicos e $\mathrm{p}=0,829$ para os fatores motivacionais).

Para os fatores higiénicos, verifica-se que o seu valor médio é superior para a existência de uma missão definida $(\mathrm{M}=2,80)$ comparativamente com a não existência $(M=2,36)$, sendo as diferenças estatisticamente não significativas $\left(\mathrm{t}_{35}=1,473, \mathrm{p}=0,150\right)$. Para os fatores motivacionais verifica-se um valor médio superior para a existência de missão definida $(\mathrm{M}=2,77)$ em comparação com a não existência $(\mathrm{M}=1,91)$, sendo as diferenças estatisticamente significativas $\left(\mathrm{t}_{35}=2,445, \mathrm{p}<0,05\right)$. 
Tabela 7

Relação entre os fatores (higiénicos e motivacionais) e o desenvolvimento pessoal e profissional: estatísticas descritivas e resultados do teste $t$

\begin{tabular}{lllllll}
\hline & $\begin{array}{l}\text { Desenvolvimento } \\
\text { pessoal e profissional }\end{array}$ & $\mathrm{N}$ & Média & $\begin{array}{l}\text { Desvio } \\
\text { Padrão }\end{array}$ & Teste t & $\mathrm{p}$ \\
\hline Fatores & Sim & 22 & 2,98 & 0,742 & $\mathrm{t} 38=2,540$ & $* 0,015$ \\
higiénicos & Não & 18 & 2,43 & 0,605 & & \\
Fatores & Sim & 22 & 3,05 & 0,728 & $\mathrm{t} 38=4,320$ & $* * * 0,000$ \\
motivacionais & Não & 18 & 2,02 & 0,767 & & \\
\hline$*$ diferença significativa para $\mathrm{p}<0,05$ & & & & & \\
$* * *$ diferença significativa para $\mathrm{p}<0,001$ & & & & &
\end{tabular}

Para avaliar a relação entre os fatores (higiénicos e motivacionais) e o desenvolvimento pessoal e profissional é utilizado o teste paramétrico $t$ de Student. Contudo, o respetivo teste é antecedido pelo teste de Levene, tendo sido verificado a existência de homocedasticidade ( $\mathrm{p}=0,391$ para os fatores higiénicos e $\mathrm{p}=0,582$ para os fatores motivacionais).

Para os fatores higiénicos, verifica-se que o seu valor médio é superior para a preocupação com o desenvolvimento pessoal e profissional do colaborador $(M=2,98)$ comparativamente com a não preocupação por parte da organização $(M=2,43)$, sendo as diferenças estatisticamente significativas $\left(\mathrm{t}_{38}=2,540, \mathrm{p}<0,05\right)$. Para os fatores motivacionais verificase um valor médio superior para a preocupação com o desenvolvimento pessoal e profissional do colaborador $(\mathrm{M}=3,05)$ em comparação com a não preocupação $(\mathrm{M}=2,02)$, sendo as diferenças estatisticamente significativas $\left(\mathrm{t}_{38}=4,320, \mathrm{p}<0,001\right)$.

\section{Tabela 8}

Relação entre os fatores (higiénicos e motivacionais) e a preocupação com a satisfação do colaborador: estatísticas descritivas e resultados do teste $\mathrm{t}$

\begin{tabular}{llllllll}
\hline & $\begin{array}{l}\text { Preocupação com a } \\
\text { satisfação do colaborador }\end{array}$ & N & Média & $\begin{array}{l}\text { Desvio } \\
\text { Padrão }\end{array}$ & Teste t & p \\
\hline Fatores & Sim & 14 & 3,13 & 0,634 & $\mathrm{t}_{37}=3,300$ & $* * 0,002$ \\
higiénicos & Não & 25 & 2,38 & 0,698 & & \\
Fatores & Sim & 14 & 3,09 & 0,674 & $\mathrm{t}_{37}=3,765$ & $* * 0,001$ \\
motivacionais & Não & 25 & 2,13 & 0,806 & & \\
\hline
\end{tabular}

**diferença significativa para $\mathrm{p}<0,01$ 
Para avaliar a relação entre os fatores (higiénicos e motivacionais) e a preocupação com a satisfação do colaborador é utilizado o teste paramétrico $t$ de Student. No entanto, o respetivo teste é antecedido pelo teste de Levene, tendo sido verificado a existência de homocedasticidade ( $\mathrm{p}=0,285$ para os fatores higiénicos e $\mathrm{p}=0,181$ para os fatores motivacionais).

Neste sentido, para os fatores higiénicos, verifica-se que o seu valor médio é superior para a preocupação com a satisfação do colaborador $(\mathrm{M}=3,13)$ em comparação com a não preocupação $(\mathrm{M}=2,38)$, sendo as diferenças estatisticamente significativas $\left(t_{37}=3,300, p<0,01\right)$. Os mesmos resultados verificaram-se para os fatores motivacionais com valor médio superior para a preocupação com a satisfação do colaborador $(\mathrm{M}=3,09)$ em comparação com a não preocupação $(M=2,13)$, sendo as diferenças também estatisticamente significativas $\left(\mathrm{t}_{37}=3,765, \mathrm{p}<0,01\right)$.

\section{Tabela 9}

Relação entre os fatores (higiénicos e motivacionais) e a partilha de resultados: estatísticas descritivas e resultados do teste $t$

\begin{tabular}{llccccc}
\hline & $\begin{array}{l}\text { Partilha de } \\
\text { resultados }\end{array}$ & N & Média & $\begin{array}{l}\text { Desvio } \\
\text { Padrão }\end{array}$ & Teste t & p \\
\hline Fatores higiénicos & Sim & 5 & 2,78 & 0,840 & $t_{36}=0,518$ & 0,608 \\
& Não & 33 & 2,59 & 0,744 & & \\
\multirow{2}{*}{ Fatores motivacionais } & Sim & 5 & 2,88 & 0,901 & $t_{36}=1,056$ & 0,298 \\
& Não & 33 & 2,42 & 0,913 & & \\
\hline
\end{tabular}

Para avaliar a relação entre os fatores (higiénicos e motivacionais) e a partilha de resultados é utilizado mais uma vez o teste paramétrico $t$ de Student. Contudo, o respetivo teste é antecedido pelo teste de Levene, tendo sido verificado a existência de homocedasticidade ( $\mathrm{p}=0,760$ para os fatores higiénicos e $\mathrm{p}=0,452$ para os fatores motivacionais).

Para os fatores higiénicos, verifica-se que o seu valor médio é superior para a partilha de resultados $(M=2,78)$ em comparação com a não partilha $(M=2,59)$, não sendo as diferenças estatisticamente significativas $\left(\mathrm{t}_{36}=0,518, \mathrm{p}=0,608\right)$. Os mesmos resultados verificaram-se para os fatores motivacionais com valor médio superior para a partilha de resultados $(M=2,88)$ em comparação com a não partilha $(M=2,42)$, não sendo as diferenças também estatisticamente significativas $\left(t_{36}=1,056\right.$, $\mathrm{p}=0,298$ ). 


\section{DISCUSSÃO DE RESULTADOS}

No que diz respeito às Hipóteses 1 e 2, os resultados encontrados permitem afirmar a existência de uma relação direta entre os fatores higiénicos/motivacionais e a motivação, permitindo a confirmação das hipóteses. Os resultados sugerem que a presença dos fatores higiénicos e motivacionais se verificam nos indivíduos motivados, embora estes fatores sejam independentes, tal como descrito pela Teoria dos Dois Fatores de Herzberg. Em Portugal foram realizados estudos semelhantes que corroboram os resultados desta investigação, tais como os levados a cabo por Ferreira (2015), Madureira e Rodrigues (2015) e Coelho e Fontes (2012). Também no Brasil, Santos (2016), Júnior e Oliveira (2009) e Caldas e Filho (2008) levaram a cabo estudos semelhantes que corroboram os resultados desta investigação.

No que concerne às Hipóteses 3 e 4, tendo em conta os resultados obtidos, verifica-se a não existência de uma relação entre os fatores higiénicos/motivacionais e a existência de atividades de acolhimento/formação, rejeitando-se as hipóteses referidas, o que contraria a teoria anteriormente enunciada. Quanto às Hipóteses 5 e 6 verifica-se que a definição da missão está positivamente relacionada com os fatores motivacionais, corroborando a hipótese 6 e rejeitado a hipótese 5. Considerando a definição da missão como uma forma de orientação do progresso e desenvolvimento que integram os fatores motivacionais, estes resultados vão ao encontro do enunciado pela Teoria dos Dois Fatores. Tal como verificado por Rodrigues, Neto e Filho (2014) a definição da missão organizacional contribui efetivamente para a motivação do colaborador corroborando os resultados apresentados.

Relativamente às Hipóteses 7 e 8 , tendo em consideração os resultados obtidos, verifica-se a existência de uma relação direta entre os fatores higiénicos/motivacionais e o desenvolvimento pessoal e profissional, corroborando as hipóteses referidas. Tendo em conta a teoria anteriormente enunciada, o desenvolvimento pessoal e profissional estão relacionados apenas com os fatores motivacionais, fatores passiveis de ser verificados apenas quando se verificam os fatores higiénicos, pelo que os resultados corroboram a teoria apresentada. Baiocchi e Magalhães (2004) verificaram uma relação positiva entre o comprometimento com a carreira (desenvolvimento profissional) e a motivação, o que confirma, em parte, os resultados apresentados. Tendo em conta as Hipóteses 9 e 10, com base nos resultados, verifica-se a existência de uma relação direta entre os 
fatores higiénicos/motivacionais e a satisfação do colaborador, pelo que se verifica as hipóteses referidas. Tendo em conta a teoria anteriormente enunciada, os fatores motivacionais são supostos causadores de satisfação, quando estão presentes, embora não sejam causadores de insatisfação quando estão ausentes. Da mesma forma, a presença dos fatores higiénicos não gera motivação, mas a sua ausência gera insatisfação. Neste sentido, os resultados apresentados corroboram, mais uma vez, a teoria referida. As investigações levadas a cabo por Júnior (2001) e Costa (2012) relativamente à relação entre satisfação e motivação no trabalho corroboram os resultados apresentados neste estudo.

Por último, no que concerne às Hipótese 11 e 12, verifica-se, com base nos resultados obtidos, a não existência de uma relação entre os fatores higiénicos/motivacionais e a partilha de resultados, pelo que se rejeita as referidas hipóteses. Considerando a partilha de resultados como uma componente extra do salário, é contrariada a teoria enunciada, uma vez que o salário integra os fatores higiénicos.

\section{CONCLUSÃO}

A motivação do colaborador permite à organização obter ganhos de produtividade, eficiência e eficácia, pelo que tem vindo a assumir uma importância crescente no contexto atual como forma de melhorar o desempenho (Nascimento, 2008). Tendo em conta esta importância têm vindo a surgir diversas teorias e investigações que exploram esta temática.

$\mathrm{Na}$ atualidade, a motivação do colaborador é mais urgente do que nunca. Trata-se de um imperativo do ponto de vista de desempenho, pelo que dispor de colaboradores motivados permite às organizações desenvolverem-se e crescerem de forma sustentada. No entanto, o processo de motivação é complexo e variado, sendo a sua mensuração impossível do ponto de vista direto, apenas possível através de uma análise aprofundada dos fatores ou variáveis quantitativas e qualitativas que possam influenciar a motivação (Ramos, 2009).

Esta preocupação com a motivação dos colaboradores não é recente. Ao longo do tempo as organizações têm vindo a preocupar-se com a motivação dos seus colaboradores. Atualmente, esta preocupação tem vindo a tornar-se cada vez mais visível. Esta tendência tem vindo a incentivar a investigação científica relativa a este tema e o 
desenvolvimento de novas teorias no contexto organizacional (Pinto, 2007).

Com base neste estudo foi possível verificar a aplicabilidade da Teoria dos Dois Fatores proposta por Herzberg na sociedade portuguesa, uma vez que se verifica uma relação direta entre os fatores higiénicos ou motivacionais com a motivação do colaborador, a definição da missão, o desenvolvimento pessoal e profissional e a satisfação do colaborador, apesar de se verificarem algumas peculiaridades, tais como não ter sido verificada a existência da mesma relação com as atividades de acolhimento/formação e a partilha de resultados.

Este estudo teve como limitação a utilização de apenas uma teoria na investigação empírica, bem como os dados daí resultantes serem transversais, impedindo-se a análise da evolução de algumas variáveis não estáticas, dada a natureza dinâmica da temática em análise. Por último, também a dimensão da amostra constitui uma limitação da investigação.

Para futuras investigações é proposta a realização de estudos semelhantes que visem testar a aplicabilidade de outras teorias, bem como confirmar ou refutar os resultados e contributos deste artigo contribuindo para a evolução do conhecimento científico desta temática.

\section{REFERÊNCIAS BIBLIOGRÁFICAS}

Andrade, C. R., Pereira, L. Z., \& Ckagnazaroff, I. B. (2007). Elementos de satisfação e insatisfação no trabalho operacional: revisitando Herzberg. Revista Gestão \& Tecnologia, 7(1), 67-89. Recuperado de: http://revistagt.fpl.edu.br/get/article/view/189

Baiocchi, A. C., \& Magalhães, M. (2004). Relações entre processos de comprometimento, entrincheiramento e motivação vital em carreiras profissionais. Revista Brasileira de Orientação Profissional, 5(1), 6369. Recuperado de:

http://pepsic.bvsalud.org/scielo.php?script=sci_arttext\&pid=S167933902004000100006

Bergamini, W., \& Bullet, C. (1990). Motivação: mitos, crenças e malentendidos. Revista de Administração de Empresas, 30(2), 23-34. Recuperado de: http://www.scielo.br/scielo.php?pid=S003475901990000200003\&script=sci_arttext

Boas, A. A. V., Esteves, R. C., \& Ferreira, A. (2006). Teorias de Motivação: um estudo de caso sobre a percepção das lideranças. 
Simpósio de Engenharia de Produção (SIMPEP). Recuperado de: https://www.aedb.br/seget/arquivos/artigos1 1/52614580.pdf

Bueno, M. (2002). As teorias de motivação humana e sua contribuição para a empresa humanizada: um tributo a Abraham Maslow. Revista do Centro de Ensino Superior de Catalão, 6. Recuperado de : http://sinop.unemat.br/site_antigo/prof/foto_p_downloads/fot_10529a s_teobias_de_motivayyo_humana_e_sua_contbibuiyyo_paba_a_empb esa_humanizada_pdf.pdf

Caldas, R. K. P., \& Filho, A. (2008). Fatores motivacionais determinantes do vínculo com o trabalho: o caso de uma instituição de ensino superior. Revista da FARN, 6, 53-67. Recuperado de: http://www.revistaunirn.inf.br/revistaunirn/index.php/revistaunirn/arti cle/viewFile/121/135

Cidade, M. M. A. C. C. (2013). A motivação em época de crise: uma investigação interpretativista sobre a motivação de profissionais em Portugal durante a crise pós 2008. (Tese de mestrado não publicada). Universidade Católica Portuguesa, Lisboa. Recuperado de: https://repositorio.ucp.pt/handle/10400.14/13412

Coelho, S. D. A., \& Fontes, F. O. (2012). Fatores Higiénicos e Motivadores dos Funcionários Públicos: um estudo de caso com recurso à teoria dos dois fatores de Herzberg. (Tese de Mestrado não publicada). Instituto Superior Miguel Torga, Coimbra. Recuperado de: http://repositorio.ismt.pt/handle/123456789/326

Cortinhas, A. (2014). Motivação e satisfação no trabalho em contextos sociais e económicos diferentes - crise e crescimento. (Tese de Mestrado não publicada). Instituto Universitário de Lisboa, Lisboa. Recuperado de: https://repositorio.iscte-iul.pt/handle/10071/10252

Costa, M. M. D. S. B. (2012). Motivação e Satisfação na Força Aérea Portuguesa (Tese de doutoramento não publicada). Instituto Superior de Economia e Gestão, Lisboa. Recuperado de: https://www.repository.utl.pt/handle/10400.5/4417

Cunha, M. P., Rego, A., Cunha, R. C., \& Cardoso, C. C. (2007). Manual de comportamento organizacional e gestão. Lisboa: Editora RH.

Eccheli, S. D. (2008). A motivação como prevenção da indisciplina. Educar Em Revista, 32, 199-213. Recuperado de: http://www.redalyc.org/html/1550/155013363014/

Ferreira, A., BOAS, A. A. V., Esteves, R., Fuerth, L. R., \& Silva, S. D. (2006). Teorias de motivação: uma análise da percepção das lideranças sobre suas preferências e possibilidade de complementaridade. SIMPEP-Bauru, 6. Recuperado de:

http://www.simpep.feb.unesp.br/anais/anais_13/artigos/114.pdf 
Ferreira, T. M. S. (2015). Os fatores motivacionais na gestão da força de vendas e a sua influência no desempenho individual. (Tese de doutoramento não publicada) Universidade Fernando Pessoa, Porto. Recuperado de: https://bdigital.ufp.pt/handle/10284/5083

Fraga, L. D. (2005). Motivação nas organizações. Revista da Católica, 3(5). Recuperado de:

http://www.catolicaonline.com.br/revistadacatolica2/artigosv3n5/artig o05.pdf

Figueiredo, L. J. L. (2012). A gestão de conflitos numa organização e consequente satisfação dos colaboradores. (Tese de mestrado não publicada). Universidade Católica Portuguesa, Viseu. Recuperado de: https://repositorio.ucp.pt/handle/10400.14/8865

Galhanas, C. R. G. (2009). A motivação dos recursos humanos nos novos modelos de gestão da administração pública. (Tese de mestrado não publicada). Instituto Superior de Economia e Gestão, Lisboa. Recuperado de: https://www.repository.utl.pt/handle/10400.5/1757

Gawel, J. E. (1997). Herzberg's theory of motivation and Maslow's hierarchy of needs. Practical Assessment, Research \& Evaluation, 5(11). Recuperado de:

$\mathrm{http} / /$ pareonline.net/getvn.asp?v=5\&n=11\&iforg_highlight=Computer

Herzberg, F., Mausner, B., \& Snyderman, B.B. (1959). The Motivation to work. New Yourk: Transaction Publishers.

Júnior, N. S. (2001). Satisfação no trabalho: um estudo entre os funcionários dos hotéis de João Pessoa. Periódicos Eletrônicos de Psicologia, 6(1), 47-57. Recuperado de:

http://pepsic.bvsalud.org/scielo.php?script=sci_arttext\&pid=S141382712001000100007

Júnior, P. E. B., \& Oliveira, J. D. (2009). Motivação no trabalho: avaliando o ambiente organizacional. Congresso Nacional de Excelência em Gestão: Gestão do Conhecimento para a Sustentabilidade.

Lima, M. L., Vala, J., \& Monteiro, M. B. (1988). Os determinantes da satisfação organizacional: confronto de modelos. Análise psicológica, 6(3), 441-457. Recuperado de: http://repositorio.ispa.pt/handle/10400.12/2224

Lobos, J. (1975). Teorias sobre a motivação no trabalho. Revista de Administração de empresas, 15(2), 17-25. Recuperado de: http://www.scielo.br/scielo.php?pid=S003475901975000200002\&script=sci_arttext 
Madureira, C., \& Rodrigues, M. (2015). Fatores de motivação dos trabalhadores na Administração Pública Central em Portugal. Revista de Administração e Emprego Público, 83-110. Recuperado de: https://www.dgaep.gov.pt/upload/Estudos/fatores_motivacao_finalnov.pdf

Marôco, J. (2011). Análise Estatística com o SPSS Statistics. (5.ed.). Lisboa: Edições ReportNumber.

Nascimento, E. (2008). Comportamento Organizacional. Curitiba: IESDE. Recuperado de:

https://pt.scribd.com/document/251170662/O-Processo-Motivacionalpdf

Oliveira, M. J. N. O. (2010). Estudo de caso sobre os fatores motivacionais em agências do banco brasileiro. (Tese de mestrado não publicada) Universidade Federal do Rio Grande do Sul, Porto Alegre. Recuperado de:

http://www.lume.ufrgs.br/handle/10183/30301

Pedroso, B., \& Pilatti, L. A. (2010). Revisão literária dos modelos clássicos de avaliação da qualidade de vida no Trabalho: um debate necessário. In Qualidade de vida: evolução dos conceitos e práticas no século XXI. (pp.197-206). Campinas: IPES Editorial. Recuperado de:

https://www.fef.unicamp.br/fef/sites/uploads/deafa/qvaf/evolucao_cap 21.pdf

Pedroso, B., Pilatti, L. A., Santos, C. B., \& Junior, G. S. (2010). Potencial motivador do trabalho: tradução e adaptação cultural do instrumento de Hackman e Oldham. Revista Produção Online, 10(3), 670-697. Recuperado de: https://producaoonline.org.br/rpo/article/view/533/719

Pérez-Ramos, J. (1990). Motivação no trabalho: abordagens teóricas. Psicologia USP, 1(2), 127-140, Recuperado de: http://pepsic.bvsalud.org/scielo.php?script=sci_arttext\&pid=S167851771990000200004

Pinto, E. P. (2007). Insatisfação com sistemas organizacionais e repercussão no atendimento a clientes. Comportamento Organizacional e Gestão, 13(2), 261-281. Recuperado de: http://repositorio.ispa.pt/bitstream/10400.12/5005/1/COG\%2C $13 \% 282 \% 29 \% 2 \mathrm{C} 261-281 . p d f$

Ramos, F. S. (2009). A influencia da liderança na motivação: um estudo sobre o programa de trainees 2008 da Galp energia. (Tese de mestrado não publicada) Instituto Universitário de Lisboa, Lisboa. Recuperado de: https://repositorio.iscte-iul.pt/handle/10071/1732 
Rodrigues, W. A., Reis Neto, M. T., \& Filho, C. G. (2014). As influências na motivação para o trabalho em ambientes com metas e recompensas: um estudo no setor público. Revista Brasileira de Administração Pública, 48(1), 253-273. Recuperado de: http://www.scielo.br/scielo.php? script=sci_arttext\&pid=S0034$76122014000100011 \& \operatorname{lng}=\mathrm{en} \& n r m=$ iso

Sampaio, J. R. (2009). O Maslow desconhecido: uma revisão de seus principais trabalhos sobre motivação. Revista de Administração RAUSP, 44 (1), 5-16. Recuperado de: http://www.redalyc.org/html/2234/223417526001/

Santos, R. (2016). Motivação no ambiente de trabalho: Uma aplicação da teoria dos dois fatores de Frederick Herzberg $O$ caso da empresa Rossi Residencial. (Tese de mestrado não publicada). Pontifícia Universidade Católica, Rio de Janeiro. Recuperado de: https://www.maxwell.vrac.puc-rio.br/30511/30511.PDF

Spink, M. J. P. (2007). Pesquisando no cotidiano: recuperando memórias de pesquisa em Psicologia Social. Revista Psicologia \& Sociedade, 19(1). Recuperado de: http://www.scielo.br/pdf/\%0D/psoc/v19n1/a02v19n1.pdf

Tadin, A. P., Rodrigues, J. A. E., Dalsoquio, P., Guabiraba, Z. R., \& Miranda, I. T. P. (2006). O conceito de motivação na teoria das relações humanas. Maringá Management, 2(1). Recuperado de: http://www.maringamanagement.com.br/novo/index.php/ojs/article/vi ew/36/19

Tureta, C., Rosa, A. R., \& Ávila, S. C. (2006). Da teoria sistêmica ao conceito de redes interorganizacionais: um estudo exploratório da teoria das organizações. Revista de Administração da Unimep, 4(1), 1-16. Recuperado de:

http://www.spell.org.br/documentos/ver/26494/da-teoria-sistemica-aoconceito-de-redes-interorganizacionais--um-estudo-exploratorio-dateoria-das-organizacoes 